\title{
VALIDAÇÃO DE ESCALA DE DETERMINAÇÃO FUNCIONAL DA QUALIDADE DE VIDA NA ESCLEROSE MÚLTIPLA PARA A LÍNGUA PORTUGUESA
}

\author{
Maria Fernanda Mendes', Silvia Balsimelli3, \\ Gabriela Stangehaus4, Charles Peter Tilbery²
}

\begin{abstract}
RESUMO - O objetivo deste estudo é traduzir para o português, realizar a equivalência cultural e validar a Escala de Determinação Funcional de Qualidade de Vida (DEFU). Foi realizado estudo longitudinal, com 143 pacientes com esclerose multipla (EM) clinicamente definida. A validação da escala foi determinada pela correlação dos escores do DEFU com a Escala de Incapacidade Funcional Expandida (EDSS) e a Escala Ambulatorial (EAN). A consistência interna (alfa) foi semelhante à observada originalmente. Observamos haver correlação entre as subescalas $(0,35-0,71)$ e destas com o EDSS e o EAN. O coeficiente Kendall Tau evidenciou haver associação significante entre a visita inicial e a de três meses. A sensibilidade da escala foi testada considerando dois grupos de pacientes: com EDSS $<3,5$ e com EDSS $\geq 3,5$. Observamos escores maiores nos pacientes com EDSS mais baixo, quando comparados com aqueles com comprometimento moderado. Baseados neste estudo, concluímos que esta versão do questionário DEFU traduzido para a língua portuguesa preenche os critérios de confiabilidade, sensibilidade e validade.
\end{abstract}

PALAVRAS-CHAVE: esclerose múltipla, qualidade de vida, escala de avaliação.

\begin{abstract}
Validation of the functional assessment of multiple sclerosis quality of life instrument in a Portuguese language

ABSTRACT - The objective of the present study was to translate, to do cultural equivalence and validation of the Functional Assessment of Multiple Sclerosis quality of life instrument (FAMS) to Portuguese. The database analyzed is longitudinal, with 143 patients with clinically defined multiple sclerosis (MS). The validity of the scale was established by correlating total FAMS scores and sub-scales scores to EDSS and EAN. The internal consistency reliability were similar to those of the original FAMS. All sub-scales correlated with each other (0.35 to 0.71). Total FAMS score correlated significantly with EDSS, and EAN. All sub-scales correlated to EDSS. Kendall's Tau coefficient, showed significant associations between basal and first visit. Responsiveness was investigated considering two groups: patients with EDSS $<3.5$ and with EDSS $\geq 3.5$. There was significantly higher score in the group with light EDSS when compared to the group of patients with moderate EDSS. One can conclude, based on this retrospective study that FAMS instrument translated to Brazilian Portuguese can be considered reliable, responsive and content valid.
\end{abstract}

KEY WORDS: multiple sclerosis, quality of life, measurement scale.

A esclerose múltipla (EM) é doença crônica e progressiva, que acomete adulto jovem, com idade inferior aos 40 anos e leva ao aparecimento de diversos sintomas e sinais neurológicos, muitas vezes incapacitantes ${ }^{1}$. A Escala de Incapacidade Funcional Expandida (EDSS) ${ }^{2}$ é considerada como a medida primária de eficácia na grande maioria dos ensaios clínicos, embora supervalorize a mobilidade e seja pouco sensível aos outros aspectos da doença ${ }^{3}$. A avaliação da qualidade de vida (QoL) permite analisar o impacto da doença e dos tratamentos propostos sob a perspectiva do doente ${ }^{4}$. $\mathrm{O}$ conceito de QoL relacionado à saúde do indivíduo definido pela Constituição da Organização Mundial de Saúde se confundia com o próprio conceito de saúde, definido como "...um estado de completo bem estar físico, mental e social, e não apenas a ausência de doença" ${ }^{\prime \prime}$. Em 1993, esta definição foi

Centro de Atendimento e Tratamento de Esclerose Múltipla (CATEM) da Disciplina de Neurologia do Departamento de Medicina da Faculdade de Ciências Médicas da Santa Casa, São Paulo SP Brasil: 'Professor Instrutor; ${ }^{2}$ Professor Adjunto; ${ }^{3}$ Psicóloga; ${ }^{4}$ Professora Adjunta Disciplina de Estatística da Universidade Estadual de Campinas (UNICAMP), Campinas SP, Brasil.

Recebido 2 Maio 2003, recebido na forma final 8 Agosto 2003. Aceito 15 Setembro 2003.

Dra. Maria Fernanda Mendes - Rua Itacolomi 333/112 - 01239-020 São Paulo SP - Brasil. E-mail: mendesnovo@uol.com.br. 
revista e ampliada, abrangendo valores mais amplos. Hoje a qualidade de vida relacionada à saúde é definida como "...a percepção do indivíduo quanto à sua situação no contexto da sua cultura e dos valores da sociedade onde vive, e em relação aos seus objetivos, expectativas, padrões e interesses..." ${ }^{\prime 6}$. Fica claro por esta definição que o conceito de qualidade de vida é multidimensional, e incorpora a percepção do indivíduo sobre diversos aspectos da vida?.

Para avaliação da QoL é fundamental a utilização de instrumentos adequados, que sejam de fácil aplicação e quantificáveis, contemplando os aspectos subjetivos da vida do paciente, como suas expectativas, percepções e emoções; mensurando a repercussão da doença e não a incapacidade. A escala ideal deverá ter sensibilidade, especificidade e ser de fácil reprodução. É fundamental que, por melhor que seja o instrumento, ele esteja adaptado às condições culturais e seja validado para o idioma do país onde será utilizado ${ }^{8}$. Vários questionários específicos foram desenvolvidos para avaliar a QoL na EM, não havendo consenso quanto ao melhor questionário. Estes instrumentos avaliam o impacto da doença, estando mais relacionados à incapacidade e aos sintomas próprios da doença. Os questionários gerais, por sua vez, medem a qualidade de vida no seu todo, porém não são sensíveis em situações peculiares de cada doença e para avaliar os efeitos das terapêuticas utilizadas ${ }^{4}$. Entre os diversos questionários específicos para EM, destacase o Functional Assessment of Multiple Sclerosis (FAMS) ${ }^{9}$, que inclui aspectos funcionais e diversas variáveis de qualidade de vida. Este instrumento vem sendo utilizado em ensaios clínicos por permitir a abordagem de amplo espectro de sintomas, dificuldades e alterações psicossociais associadas com a $\mathrm{EM}^{10}$.

O questionário MOS-36, considerado um instrumento geral para a avaliação de QoL ${ }^{11}$, foi validado no Brasil por Ciconelli em 1997², porém, não há nenhum questionário específico para esclerose múltipla que possa ser utilizado em estudos longitudinais. O objetivo deste estudo é validar o FAMS para a língua portuguesa.

\section{MÉTODO}

Instrumento - O Functional Assessment of Multiple Sclerosis (FAMS) foi desenvolvido por Cella e col, em 1996 como uma escala específica para quantificar o estado de saúde dos pacientes com esclerose múltipla ${ }^{9}$. Esta escala, traduzida para a língua portuguesa como Escala de Determinação Funcional da Qualidade de Vida em pa- cientes com Esclerose Múltipla (DEFU) foi adaptada para a língua portuguesa em nosso Serviço em 1996.

Utilizaram como base um questionário genérico desenvolvido para pacientes com câncer ${ }^{9}$, adicionando questões sobre sintomas e problemas relacionados à EM. A versão original, em inglês, é composta de 59 itens, sendo 44 deles utilizados para a obtenção do escore. Os 15 itens restantes são apresentados por fornecer informações clínicas e sociais relevantes, porém não devem ser utilizados para a obtenção do escore final ${ }^{9}$. Em sua forma final, a DEFU é composta por 6 subitens válidos para análise: mobilidade (7 itens), sintomas ( 7 itens), estado emocional (7 itens), satisfação pessoal (7 itens), pensamento e fadiga (9 itens) e situação social e familiar (7 itens). $\mathrm{O}$ formato das respostas permite escores de 0 a 4 para cada item, no formato tipo Likert, sendo considerado o escore reverso para as questões construídas de forma negativa. Desta forma, os escores maiores refletem melhor qualidade de vida. As cinco subescalas com 7 itens permitem escores de 0 a 28 e a subescala com 9 itens (pensamento e fadiga) tem seus escores variando de 0 a 36 . O escore total da FAMS varia de 0 a $176[5(28)+1(36)]^{9}$.

Para a sua validação, a escala DEFU foi traduzida para a língua portuguesa por duas pessoas, bilíngües, uma delas tendo o inglês como língua materna. A escala foi posteriormente vertida para o inglês, chegando-se a um resultado final de consenso. $O$ formato final da escala foi mantido, com 6 subitens e 44 questões válidas para obtenção do escore. Um estudo piloto realizado com 20 pacientes não demonstrou dificuldades de entendimento das questões. Visando reduzir o tempo necessário para a aplicação da escala, as questões não utilizadas para o escore final e apresentadas no anexo foram reduzidas para nove. Para contornar o problema de baixa escolaridade, o examinador permanece presente durante a aplicação do questionário. A versão traduzida encontrase no Anexo 1.

O questionário foi aplicado na visita inicial e após três meses. No momento das visitas clínicas foram aplicadas duas escalas de incapacidade funcional, a Escala de Incapacidade Funcional Expandida de Kurtzke² (EDSS) e a Escala Ambulatorial ${ }^{13}$ (EAN), para posterior análise de validade e de sensibilidade.

Pacientes - o questionário foi aplicado em 143 pacientes com EM, definida pelos critérios de Poser e col. ${ }^{14}$, atendidos no Centro de Atendimento e Tratamento de Esclerose Múltipla da Faculdade de Ciências Médicas da Santa Casa de São Paulo (CATEM). Foram incluídos no estudo pacientes com EDSS variando de 0 a 6,0 e com escolaridade superior a 4 anos. Os pacientes são provenientes principalmente de áreas urbanas, porém o centro recebe pacientes de toda a região metropolitana de São Paulo. A pesquisa foi aprovada delo Comitê de Ética Médica da Faculdade de Ciências Médicas de Santa Casa de São Paulo.

Método estatístico - a validação de um instrumento 
Nunca Um pouco

Às vezes

Muitas vezes

Sempre

\section{Mobilidade}

1. Tenho problemas, devido a minha condição física, em manter minha família

2. Sou capaz de trabalhar mesmo em casa

3. Tenho problemas para andar

4. Tenho limitações na vida social

5. Minhas pernas são fortes

6. Tenho constrangimento em lugares públicos

7. Fiz planos por causa de minha doença

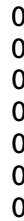

\section{Sintomas}

8. Tenho náuseas

9. Tenho dores

10. Sinto-me doente

11. Sinto-me fraco

12. Tenho dores nas juntas

13. Tenho dores de cabeça

14. Tenho dores musculares

Estado emocional

15. Estou triste

16. Estou perdendo a fé na luta contra minha doença

17. Sou capaz de curtir a vida

18. Sinto-me prisioneiro da minha doença

19. Estou deprimido por causa da minha situação

20. Sinto-me inútil

21. Sinto-me dominado pela doença

\section{Satisfação pessoal}

22. Meu trabalho mesmo em casa me satisfaz

23. Aceitei minha doença

24. Tenho prazer no que faço quando me divirto

25. Estou satisfeito com a minha qualidade de vida

26. Estou frustrado por causa da minha condição

27. Sinto um propósito na vida

28. Sinto-me motivado em realizar coisas

\section{Pensamento e fadiga}

29. Tenho perda de energia

30. Sinto-me cansado

31. Tenho dificuldade em iniciar tarefas por estar cansado

32. Tenho dificuldade em terminar tarefas por estar cansado

33. Preciso repousar durante o dia

34. Tenho dificuldade em lembrar das coisas

35. Tenho dificuldade em me concentrar

36. Meu raciocínio está lento

37. Tenho dificuldade em aprender novas tarefas

\section{Situação social e familiar}

38. Sinto-me distante dos amigos

39. Tenho suporte emocional da família

40. Tenho suporte dos amigos e vizinhos

41. Minha família aceitou a doença

42. A comunicação da família a respeito da doença é pobre

43. Minha família tem dificuldades em reconhecer minha piora

44. Sinto-me excluído dos fatos

\section{Anexo}

45. Os efeitos colaterais me incomodam

46. Sou forçado a passar algum tempo na cama

47. Sinto-me junto ao parceiro

48. Tive contato sexual no último ano. Não...Sim...

Se sim, estou satisfeito com minha vida sexual

49. A equipe médica é acessível às minhas dúvidas

50. Estou orgulhoso de como enfrento a doença

51. Sinto-me nervoso

52. Estou preocupado que minha doença piore

53. Estou dormindo bem

$\begin{array}{llll}1 & 2 & 3 & 4 \\ 1 & 2 & 3 & 4 \\ 1 & 2 & 3 & 4 \\ 1 & 2 & 3 & 4 \\ 1 & 2 & 3 & 4 \\ 1 & 2 & 3 & 4 \\ 1 & 2 & 3 & 4\end{array}$

Escore parcial:

$\begin{array}{llll}1 & 2 & 3 & 4 \\ 1 & 2 & 3 & 4 \\ 1 & 2 & 3 & 4 \\ 1 & 2 & 3 & 4 \\ 1 & 2 & 3 & 4 \\ 1 & 2 & 3 & 4 \\ 1 & 2 & 3 & 4\end{array}$

Escore parcial:

\begin{tabular}{llll}
1 & 2 & 3 & 4 \\
1 & 2 & 3 & 4 \\
1 & 2 & 3 & 4 \\
1 & 2 & 3 & 4 \\
1 & 2 & 3 & 4 \\
1 & 2 & 3 & 4 \\
1 & 2 & 3 & 4 \\
\hline
\end{tabular}

$\begin{array}{llll}1 & 2 & 3 & 4 \\ 1 & 2 & 3 & 4 \\ 1 & 2 & 3 & 4 \\ 1 & 2 & 3 & 4 \\ 1 & 2 & 3 & 4 \\ 1 & 2 & 3 & 4 \\ 1 & 2 & 3 & 4\end{array}$

$\begin{array}{ccc}2 & 3 & 4 \\ 2 & 3 & 4 \\ 2 & 3 & 4 \\ 2 & 3 & 4 \\ 2 & 3 & 4 \\ 2 & 3 & 4 \\ 2 & 3 & 4 \\ 2 & 3 & 4 \\ 2 & 3 & 4\end{array}$

\begin{tabular}{llll}
1 & 2 & 3 & 4 \\
1 & 2 & 3 & 4 \\
1 & 2 & 3 & 4 \\
1 & 2 & 3 & 4 \\
1 & 2 & 3 & 4 \\
1 & 2 & 3 & 4 \\
1 & 2 & 3 & 4 \\
\cline { 2 - 3 } & Escore parcial: & & \\
\hline & 2 & 3 & 4 \\
1 & 2 & 3 & 4 \\
1 & 2 & 3 & 4 \\
& 2 & & 4 \\
1 & 2 & 3 & 4 \\
1 & 2 & 3 & 4 \\
1 & 2 & 3 & 4 \\
1 & 2 & 3 & 4 \\
1 & 2 & 3 & 4 \\
1 & 2 & 3 &
\end{tabular}

Escore total: 
de qualidade de vida requer a avaliação dos dados obtidos com a aplicação do instrumento a pacientes, quanto à consistência interna, confiabilidade ou estabilidade do instrumento, validade e sensibilidade ${ }^{8}$.

A consistência interna foi medida usando-se o coeficiente alfa de Cronbach, que mede constructos latentes determinando a consistência interna dos itens através da correlação média das questões dentro de um item. Quanto maior o coeficiente alfa, mais este contribui na construção da escala, sendo considerados bons os valores entre 0,65 e 1 . A sensibilidade da escala foi avaliada subdividindo-se os pacientes em dois grupos, de acordo com o valor do EDSS: aqueles com EDSS entre 0 e 3,0 apresentam sintomas leves e aqueles com EDSS acima de 3,5 apresentam sintomas moderados. Os escores médios nos dois grupos foram comparados.

A confiabilidade do instrumento foi obtida usando o coeficiente Tau de Kendall. Está baseado no número de pares de observação concordantes e discordantes, fornecendo valores entre -1 e 1 , sendo a confiabilidade tanto maior quanto maior for o coeficiente Tau. Para a medida de teste-reteste, foram consideradas a medida inicial e a primeira medida que ocorria após três meses.

A validade do questionário foi medida usando-se o coeficiente de correlação de Pearson, o que permitiu avaliar o comportamento desta escala em função de outras medidas de avaliação clínica, como o EDSS, o EAN.

\section{RESULTADOS}

Após a tradução e determinação da versão final em português da escala DEFU, foram avaliados longitudinalmente 143 pacientes com EM. A média de idade dos pacientes era 34,8 $\pm 8,1$ anos, com escolaridade média de 10,8 $\pm 4,7$ anos, sendo $78,1 \%$ do sexo feminino, com duração média da doença de $69,4 \pm 63,8$ meses. As médias do EDSS e EAN eram $2,19 \pm 1,65$ e $8,41 \pm 9,69$ respectivamente. Os es-
Tabela 1. Análise da consistência interna da Escala de Determinação Funcional da Qualidade de Vida na Esclerose Múltipla (DEFU).

\begin{tabular}{lcc}
\hline DEFU & $\begin{array}{c}\text { Média } \pm \text { DP } \\
(n=143)\end{array}$ & $\begin{array}{c}\text { Coeficiente } \\
\text { alfa de } \\
\text { Cronbach }\end{array}$ \\
\hline Mobilidade & $18,28 \pm 5,84$ & 0,74 \\
Sintoma & $20,08 \pm 6,06$ & 0,82 \\
Estado emocional & $22,54 \pm 5,80$ & 0,87 \\
Satisfação pessoal & $20,39 \pm 6,36$ & 0,80 \\
Pensamento e fadiga & $22,95 \pm 8,60$ & 0,81 \\
Situação Social/familiar & $21,60 \pm 6,13$ & 0,76 \\
DEFU total & $125,96 \pm 29,50$ & 0,86 \\
\hline
\end{tabular}

DP, desvio padrão

cores médios e desvios padrões obtidos nos 143 pacientes, nas seis subescalas e o total encontram-se na Tabela 1. A consistência interna, avaliada pelo coeficiente alfa de Cronbach, foi elevado em todas as subescalas, demonstrando a homogeneidade do teste após a tradução para a língua portuguesa (Tabela 1).

A confiabilidade do instrumento (teste-reteste) foi avaliada em 90 pacientes, sendo utilizada a medida basal e aquela obtida após três meses. $\mathrm{O}$ coeficiente Tau de Kendall mostrou-se elevado, com escores variando de 0,35 a 0,65. Os menores valores foram obtidos na subescala estado emocional. Houve associação significante $(p<0,001)$ em todos os escores das subescalas e no escore total obtidos nas duas visitas (Tabela 2).

A validade da escala na língua portuguesa foi testada através de sua correlação com outras me-

Tabela 2. Análise da confiabilidade (teste-reteste) da Escala de Determinação Funcional da Qualidade de Vida (DEFU)

\begin{tabular}{lccc}
\hline $\begin{array}{l}\text { DEFU } \\
(\mathrm{n}=90)\end{array}$ & $\begin{array}{c}\text { Visita Basal } \\
\text { Média } \pm \text { DP }\end{array}$ & $\begin{array}{c}\text { Visita 1 } \\
\text { Média } \pm \text { DP }\end{array}$ & Kendall-Tau* \\
\hline Mobilidade & $18,47 \pm 5,93$ & $17,98 \pm 5,15$ & 0,544 \\
Sintoma & $20,25 \pm 5,37$ & $19,59 \pm 5,57$ & 0,421 \\
Estado emocional & $22,30 \pm 5,61$ & $23,24 \pm 5,09$ & 0,353 \\
Satisfação pessoal & $20,17 \pm 5,95$ & $21,64 \pm 5,71$ & 0,475 \\
Pensamento e Fadiga & $20,17 \pm 5,95$ & $23,87 \pm 7,39$ & 0,562 \\
Situação social/familiar & $21,37 \pm 6,15$ & $21,53 \pm 6,81$ & 0,445 \\
DEFU total & $125,87 \pm 27,14$ & $127,51 \pm 26,14$ & 0,551 \\
\hline
\end{tabular}

$\mathrm{DP}$, desvio padrão; *, dado estatisticamente significante para $\mathrm{p}<0,001$. 
Tabela 3. Coeficientes de correlação entre as Escalas DEFU, EDSS e EAN (n=143).

\begin{tabular}{lcc}
\hline DEFU & EDSS & EAN \\
\hline Mobilidade & $-0,67423^{*}$ & $0,69450^{*}$ \\
Sintoma & $-0,33157^{* *}$ & $0,31699 * *$ \\
Estado emocional & $-0,52748^{*}$ & $0,55001 *$ \\
Satisfação pessoal & $-0,43170^{*}$ & $0,38446 *$ \\
Pensamento e Fadiga & $-0,31133^{* *}$ & $0,33347^{* *}$ \\
Situação Social/familiar & $-0,35422 *$ & $0,32476 * *$ \\
DEFU total & $-0,50050^{*}$ & $0,56706 *$ \\
\hline
\end{tabular}

DEFU, determinação funcional da qualidade de vida; EDSS, escala de incapacidade funcional expandida; *, dado estatisticamente significante para $\mathrm{p}<0,0001 ; * *$, dado estatisticamente significante para $\mathrm{p}<0,0005$.

didas, como as escalas de incapacidade EDSS e EAN (Tabela 3), apresentando relação significante com o escore total e subescalas. A Tabela 4 mostra a correlação entre as subescalas e o escore total. Todas as subescalas apresentaram correlação significativa entre si, com coeficiente de associação variando entre 0,353 e 0,706 .

A sensibilidade da escala foi demonstrada através da comparação de grupos distintos. $O$ critério escolhido para a determinação dos grupos foi o escore obtido no EDSS, sendo formado dois grupos distintos, um com EDSS $<3,5(n=113)$ e outro com EDSS $\geq 3,5(n=30)$. Análises de variância foram realizadas para comparar as médias entre os grupos para cada item do DEFU e o resultado total. Com exceção do subitem sintomas há diferença estatisticamente significante entre os grupos nos demais subitens e escore total, com as médias do grupo com EDSS < 3,5 significativamente maior do que naqueles com EDSS $\geq 3,5$. Portanto, a versão para a língua portuguesa desta escala mostrou-se sensível para detectar mudanças em diferentes etapas da doença, demonstrando melhor qualidade de vida nos pacientes com menor incapacidade funcional (Tabela 5).

\section{DISCUSSÃO}

A DEFU é instrumento multidimensional, que permite a mensuração da qualidade de vida relacionada à saúde (HRQoL) em portadores de EM. A metodologia utilizada na sua elaboração considerou a opinião de médicos, pacientes e informações de literatura sobre QoL na EM ${ }^{9}$. Este questionário, após validação para a língua portuguesa, manteve as características daquele originalmente descrito quanto à consistência interna, confiabilidade e sensibilidade, podendo ser utilizado na prática clínica e em ensaios clínicos.

Os valores obtidos nas subescalas e no escore total na nossa população foram muito semelhantes àqueles originalmente descritos por Cella e cols no grupo de pacientes ambulatoriais e que não necessitavam de períodos de repouso durante o dia. O escore total neste grupo de pacientes foi de $129,90^{9}$ e o desta amostra 125,96. A consistência interna foi semelhante à observada por Cella e

Tabela 4. Coeficientes de correlação entre as sub-escalas da Escala de Determinação Funcional da Qualidade de Vida $(n=143)$.

\begin{tabular}{|c|c|c|c|c|c|c|}
\hline & Mobilidade & Sintomas & E. emocional & S. pessoal & Pensam/fad. & S. social/fam. \\
\hline \multirow[t]{2}{*}{ Sintoma } & 0,40431 & & & & & \\
\hline & $<, 0001$ & & & & & \\
\hline \multirow[t]{2}{*}{ E.emocional } & 0,61612 & 0,47682 & & & & \\
\hline & $<, 0001$ & $<, 0001$ & & & & \\
\hline \multirow[t]{2}{*}{ S.pessoal } & 0,53192 & 0,41913 & 0,70601 & & & \\
\hline & $<, 0001$ & $<, 0001$ & $<, 0001$ & & & \\
\hline \multirow[t]{2}{*}{ Pensam/fad. } & 0,42899 & 0,57478 & 0,51929 & 0,41130 & & \\
\hline & $<, 0001$ & $<, 0001$ & $<, 0001$ & $<, 0001$ & & \\
\hline \multirow[t]{2}{*}{ S. social/fam. } & 0,49316 & 0,35349 & 0,61400 & 0,60190 & 0,39197 & \\
\hline & $<, 0001$ & $<, 0001$ & $<, 0001$ & $<, 0001$ & $<, 0001$ & \\
\hline \multirow[t]{2}{*}{ DEFU Total } & 0,68723 & 0,53157 & 0,52748 & 0,45670 & 0,60133 & 0,35422 \\
\hline & $<, 0001$ & $<, 0001$ & $<, 0001$ & $<, 0001$ & $<, 0001$ & $<, 0001$ \\
\hline
\end{tabular}

DEFU, determinação funcional da qualidade de vida; E. emocional, estado emocional; S. pessoal, satisfação pessoal; Pensam/fad, pensamento e fadiga; S. social/fam, situação social e familiar. 
Tabela 5. Análise de sensibilidade da Escala de Determinação Funcional da Qualidade de Vida (DEFU).

\begin{tabular}{lccc}
\hline DEFU & $\begin{array}{c}\text { Média } \pm \text { DP } \\
\text { EDSS }<3,5 \\
(n=113)\end{array}$ & $\begin{array}{c}\text { Média } \pm \text { DP } \\
\text { EDSS } \geq 3,5 \\
(n=30)\end{array}$ & $p$ \\
\hline Mobilidade & $19,72 \pm 0,47$ & $12,30 \pm 0,79$ & $<0,001$ * \\
Sintoma & $20,58 \pm 0,53$ & $18,90 \pm 1,27$ & 0,173 \\
Estado emocional & $23,55 \pm 0,47$ & $18,07 \pm 1,23$ & $<0,001$ * \\
Satisfação pessoal & $21,42 \pm 0,58$ & $16,14 \pm 1,04$ & $<0,001$ * \\
Pensamento e fadiga & $24,11 \pm 0,81$ & $20,27 \pm 1,57$ & 0,031 * \\
Situação social/familiar & $22,22 \pm 0,52$ & $18,77 \pm 1,23$ & 0,004 * \\
DEFU total & $131,48 \pm 2,60$ & $104,46 \pm 5,43$ & $<0,001$ *
\end{tabular}

DP, desvio padrão; EDSS, escala de incapacidade funcional expandida; ${ }^{*}$, dado estatisticamente significante.

cols, com coeficiente alfa de Cronbach variando de 0,73 a 0,89 após a tradução e de 0,82 a 0,93 na versão da língua inglesa. Houve correlação entre as subescalas e destas com o EDSS e o EAN; os subitens mobilidade e bem estar emocional apresentaram forte correlação com o EDSS, resultados estes semelhantes aos obtidos na descrição da escala.

$\mathrm{Na}$ análise de sensibilidade, os autores avaliaram os resultados obtidos em 28 pacientes com EDSS $\leq 6,0$ e 27 com EDSS > 6,0. Encontraram-se diferenças entre os grupos apenas quanto ao subitem mobilidade, e o escore global foi semelhante. A população por nós estudada apresentava características distintas daquela originalmente descrita por Cella e cols quanto à incapacidade funcional, apresentando menores escores no EDSS. Por este motivo, utilizamos o escore $\geq 3,5$ para a comparação entre os grupos, observando diferenças significantes nos subitens mobilidade, estado emocional, pensamento/fadiga e escore total. Os autores observaram escore total médio de 113,9 para os pacientes com EDSS $\leq 6,0$ e 106,2 para aqueles com EDSS > 6,0 . Na nossa população, os escores médios foram de 131,48 quando o EDSS $<3,5$ e de 104,46 naqueles com EDSS $\geq 3,5$. Estes valores foram semelhantes àqueles observados por Cella e cols no grupo de maior incapacidade funcional, ficando demonstrada a sensibilidade do instrumento após tradução para a língua portuguesa.

Concluindo, esta validação para a língua portuguesa preenche os critérios de aplicabilidade, sensibilidade e validade de forma semelhante àque- le originalmente descrito. É um questionário que permite ao neurologista melhor conhecimento dos diversos aspectos da EM. A validação desta escala para diversos países e idiomas permitirá melhor abordagem dos pacientes e maior troca de informações entre os diversos centros de pesquisa.

\section{REFERÊNCIAS}

1. Matthews, WB. Clinical aspects. In Compston DAS (ed). McAlpine's multiple sclerosis. London: Churchill Livingstone, 1998:43-251.

2. Kurtzke, JF. Rating neurologic impairment in multiple sclerosis: an expanded disability status scale (EDSS). Neurology 1983;33:1444-1452.

3. Canadian Burden of Illness Study Group. Burden of illness of multiple sclerosis. II. Quality of Life. Can J Neurol Sci 1998;25:31-38.

4. Wiklund I Karlberg J. Evaluation of quality of life in clinical trials: selecting quality of life measures. Controlled Clinical Trials 1991,12:204S-216S.

5. World Health Organization. International classifications of impairments, disabilities and handicaps: a manual and classification relating to consequences of disease. Geneva: WHO, 1980.

6. Study Protocol for the World Health Organization project to develop a Quality of life assessment instrument (WHQOL). Quality of Life Res $1993 ; 2: 153-159$.

7. World Health Organization Quality of Life. Annotated bibliography, Rev 2 Geneva: HHO/MNH/MHP, 1998.

8 World Health Organization Quality of Life: general guidelines for methodologies on research and evaluation of traditional medicine. Geveda: WHO/EDM/TRM/2000.

9. Cella DF, Dineen $K$, Arnason B, et al. Validation of he functional assessment of multiple sclerosis quality of life instrument. Neurology 1996;47:129-139.

10. Hernández MA. Tratamiento de la esclerosis múltiple y calidad de vida. Rev Neurol 2000;30:1242-1245.

11. Ware JE, Sherbourne CD. The MOS 36-Item short-form health survey (SF-36). conceptual framework and item selection. Medical Care 1992;30:473-480.

12. Medeiros MMC. Impacto da doença e qualidade de vida dos cuidadores primários de pacientes com artrite reumatóide: adaptação cultural e validação do Caregiver Burden Scale. 1998. Tese, Universidade Federal de São Paulo, Escola Paulista de Medicina, São Paulo, Brasil. 1998.

13. Sipe JC, Knobler RL, Braheny SL, et al. A neurologic rating scale (NRS) for use in múltiple sclerosis. Neurology 1984;34:1368-1372.

14. Poser CM, Paty DW, Scheinberg L. New diagnosis criteria for multiple sclerosis: guidelines for research protocols. Ann Neurol 1983;13:227-231. 